Chapman University

Chapman University Digital Commons

Psychology Faculty Articles and Research

Psychology

$9-9-2021$

Burstiness and Stochasticity in the Malleability of Physical Activity

Vincent Berardi

David Pincus

Evan Walker

Marc A. Adams

Follow this and additional works at: https://digitalcommons.chapman.edu/psychology_articles

Part of the Behavioral Disciplines and Activities Commons, Health Psychology Commons, Other Psychiatry and Psychology Commons, and the Other Psychology Commons 


\section{Burstiness and Stochasticity in the Malleability of Physical Activity}

\section{Comments}

This is a pre-copy-editing, author-produced PDF of an article accepted for publication in Journal of Sport \& Exercise Psychology, volume 43, issue 5, in 2021 following peer review. The definitive publisherauthenticated version is available online at https://doi.org/10.1123/jsep.2020-0340.

\section{Copyright}

Human Kinetics, Inc. 
Burstiness and Stochasticity in the Malleability of Physical Activity 


\begin{abstract}
This study examined whether patterns of self-organization in physical activity (PA) predicted long-term success in a year-long PA intervention. Increased moderate-to-vigorous PA (MVPA) was targeted in insufficiently active adults $(N=512)$ via goal setting and financial reinforcement. The degree to which Inverse Power Law (IPL) distributions, which are reflective of self-organization, summarized i.) daily MVPA and ii.) time elapsed between meeting daily goals (goal attainment inter-response times [IRTs]) was calculated. Goal attainment IRTs were also used to calculate burstiness, the degree to which meeting daily goals clustered in time. IPLs accurately summarized IRTs, but not daily MVPA. For participants with higher levels of MVPA early in the study, burstiness in reaching goals was associated with long-term resistance to intervention, while stochasticity in meeting goals predicted receptiveness to intervention. These results suggest that burstiness may measure self-organizing resistance to change, while PA stochasticity could be a precondition for behavioral malleability.
\end{abstract}

Key words: Behavioral Intervention; Malleability; Burstiness; Self-organization; Inverse Power Law, Exercise 


\section{INTRODUCTION}

Insufficient physical activity (PA) is a key risk factor in the most common and costly chronic health conditions, including heart disease, chronic pain, and Type 2 Diabetes (Benjamin et al., 2019). The annual combined monetary costs worldwide for these chronic health conditions is in the range of trillions of dollars (Pincus \& Guastello, 2012), with approximately $2 \%$ of all healthcare expenditures accounted for specifically by physical inactivity (Benjamin et al., 2019). Only a small percentage $(<8 \%)$ of US adults meet moderate to vigorous physical activity (MVPA) guidelines from the federal government (Troiano et al., 2008), and population levels of activity over the past two decades have not increased substantially, even with significant public health investments (Brownson, Boehmer, \& Luke, 2005; Kruger, Ham, \& Kohl III, 2005; Ward, Clarke, Nugent, \& Schiller, 2015).

Information concerning the inadequacy of physical activity is most often presented from an epidemiological perspective that aims to establish general thresholds where average risk is sufficiently reduced, which can then be recommended as targets for intervention. For example, the US Department of Health and Human Services recommends adults engage in a minimum of 150 minutes of MVPA per week to reduce all-cause mortality (U.S. Department of Health and Human Services, 2018). The health assumptions underlying this approach are clear, but the behavioral assumptions are rarely discussed, perhaps because such general targets are simple, measurable, and practical for clinicians and the general public. While benchmarks like this provide useful data-driven targets, they do not address the critical questions of individual's resistance to change. The presence of this behavioral inertia is demonstrated by a meta-analysis showing that $33 \%$ to $50 \%$ of intervention participants fail to increase PA (Dishman \& Buckworth, 1996) and other studies indicating a similar outcomes (Marcus et al., 2000; Williams et al., 2008). Resistance on a comparable scale has been identified in other domains (Silverman et al., 1996) and for clinicians charged with promoting evidence-based guidelines (Grol, 2001). In fully examining the factors that lead to this phenomenon, it will be helpful to consider self-organization theory and human behavioral dynamics to help understand the malleability/inflexibility of certain behavioral patterns in response to a known perturbation (i.e. intervention).

Self-organization is a process within complex adaptive systems whereby interactions among system components lead to the emergence of higher-level global order such as self-regulation and 
structural evolution (Kauffmann, 1995; Pincus \& Metten, 2010). Pincus and Metten have proposed a general theory of biopsychosocial resilience, which is defined as a correction in behavior towards functionality. Their framework describes biological, psychological and social systems as highly interactive network components that allow self-organizing processes to naturally arise and produce patterned outputs across these systems over time (2010). This theory posits that biopsychosocial processes facilitate shifts toward coherence (i.e., restricted experiential range and behavioral coping) under conditions of threat and toward flexibility during times of exploration and growth. Therefore, Pincus and Metten propose that the resilience of behavior in response to a perturbation (e.g., an intervention) may be functionally defined as meta-flexibility: the ability of a system to shift smoothly between coherence and flexibility, without getting stuck or decomposing (2010, p. 359). Hypothetically, this functional resilience depends upon the structural resilience of one's biopsychosocial system, defined as a network structure with optimal integrity (i.e., connectivity) and flexibility.

A variety of dynamical systems modeling strategies can be used for testing hypotheses based on self-organization and human resilience, each of which aims to measure structural integrity and flexibility within a particular biopsychosocial system (Pincus \& Metten, 2010). Since PA is a complex habit nested in time and within individuals" dynamical "life space," identifying the factors underlying resistance requires intensively longitudinal data that can reveal the potential complexity of nonlinear temporal patterns. With such data, two overlapping strategies for identifying self-organization within time-series data are most appropriate: (a) the analysis of fat-tailed or inverse power-law (IPL) distributions, which describe the exponential relationship between large and small bouts of activity (see Fig. 1); and (b) the consideration of burstiness, the degree to which bouts of activity tend to cluster together in time (Karsai, Jo, \& Kaski, 2018).

Fat-tailed IPL distributions are scale free (i.e. fractal) in nature, which make extreme events rare, but expected, outcomes if given sufficient time. This stands in contrast to normal distributions where the probabilities of extreme events rapidly decay toward zero. Self-organizing systems tend to produce fat-tailed outputs, which are ubiquitous in physiological and psychosocial health processes such as movement, balance, cardiovascular health (Goldberger, 2006; Pincus \& Metten, 2010), neurological activity (Dave, Brothers, \& Swaab, 2018), cognition (Kello, Anderson, Holden, \& Van Orden, 2008), 
self-esteem (Wong, Vallacher, \& Nowak, 2016), personality (Pincus, Cadsky, Berardi, Asuncion, \& Wann, 2019), group dynamics (Pincus, 2014), and the sequential flow of discrete individual behaviors (Pincus et al., 2014). Several studies have examined IPLs and similar "fat-tailed" distributions within the context of physical activity and health. Paraschiv-Ionescu et al (2013) found that fatter tails in activity bout length (i.e., relatively more periods of long activity) were associated with worse functionality in chronic pain subjects and Bellettiere et al. (2017) observed that fatter tails in the distribution of sedentary bouts (relatively more periods of inactivity) predicted a variety of negative health indicators. Lee et al. (2019) also found that the distribution of activity and rest times were IPLdistributed, but they did not see the expected relationship between IPL characteristics and bipolar disorder. Nakamura et al. (2007) observed that depressed individuals had fatter tails in IPL distributions of their resting period durations (i.e. relatively more long rest periods) compared to healthy individuals. They replicated these results experimentally with mice that had alterations in their daily circadian rhythms (Nakamura et al., 2008), suggesting effects are present across species. Within an intervention context, Zhang et al (2018) found that fat tails in activity patterns over time were significantly associated with improved post-treatment measures of functional movement in elderly patients with chronic pain, while more conventional PA metrics (e.g., percentage of walking and sedentary time, step counts, mean cadence) showed no such association.

\section{$<$ INSERT FIGURE 1>}

Figure 1. Gaussian (Normal) Distribution versus Pareto (Inverse Power-Law) Distribution. The Gaussian distribution stabilizes around a standardized mean $=0$ and finite variance with increasing $x$. The Pareto distribution approaches infinite mean and variance with increasing $x$, and describes fluctuations in highly interactive processes unfolding over time. Pareto distributions are scale-free, or fractal, and can be defined by an exponential relationship between size and frequency.

IPLs and other fat-tailed distributions can also be used to describe the distribution of the times between two events of interest. A common feature associated with this context is bursty activity patterns, where events occur in rapid succession over short time periods, followed by long periods of nonoccurrence (Karsai et al., 2018). Importantly, this clustering of activity within time is how inter-event IPLs emerge, meaning that bursty patterns may be considered the building blocks of fat-tailed distributions in the context of self-organization. At the level of the individual, activity becomes a selfsustaining process for the duration of the burst, rather than something that turns on/off stochastically. 
Bursty activity patterns have been observed in a variety of natural phenomena including email patterns (Vazquez, Rácz, Lukács, \& Barabási, 2007), earthquakes (Corral, 2003), and gene expression (Golding, Paulsson, Zawilski, \& Cox, 2005) and impacts the behavior of many complex processes such as network dynamics and the development of coherent structures (Delvenne, Lambiotte, \& Rocha, 2015; Eckmann, Moses, \& Sergi, 2004; Iribarren \& Moro, 2011; H. H. Jo, Perotti, Kaski, \& Kertész, 2014; Karsai et al., 2011; Starnini, Baronchelli, Barrat, \& Pastor-Satorras, 2012).

A variety of mechanisms have been proposed to account for the presence of bursty behavior patterns observed in humans. Barabasi (2005) suggested that burstiness is a result of diverse, competing stimuli and incentives that drive a prioritization among tasks, as opposed to Poisson processes that are associated with the stochastic completion of tasks, or other general heuristics like: "first-come-first served." Malmgrem et al. (2008) argued that prioritization is not necessary and that instead cyclic constraints in life cause burstiness; however Jo et al. (2012) removed known cyclic patterns from data and found that burstiness remained. Gandica et. al (2016) examined the timing of Wikipedia edits and concluded that burstiness reflected the high costs of initiating an activity versus continuing it, which they indicated could be reflective of the queuing/prioritization process suggested by Barabasi. While Sorribes et al. (2011) focused on fruit flies rather than humans, they experimentally demonstrated the role of task prioritization in producing bursty behavior.

While the task-prioritization explanation of bursty behavior does not explicitly refer to selforganization, it is a small logical step to suggest that self-organizing habits may exist within selforganizing task networks. Through this lens, PA habits potentially exist within a relatively complex decision-making matrix involving competition or cooperation with other potential behavioral options that could either interfere with or facilitate the uptake of exercise. For example, adopting a dog could enable increases in PA (Cutt, Knuiman, \& Giles-Corti, 2007), while taking on a new office job could interfere (Blackwell \& Clarke, 2016). For individuals with many interacting task priorities, one may expect higher burstiness and fatter tails in PA patterns, to the degree that a decision to exercise versus engaging in other activities represents a task queuing process. By contrast, other people may have relatively few entanglements with PA, and thus should produce more stochastic intervals between bouts 
of PA, and more exponential distributions. Importantly, the tangled stimuli and incentives need not be overt like a dog or new job, but may be covert - comprised of physiological states, moods, attitude, and/or interpersonal contexts. As such, they may be highly individualized, and difficult to fully assess at the start of a PA intervention.

The present secondary analysis investigated whether indicators of PA self-organization were present and associated with resistance to behavior change within a PA intervention that measured daily MVPA using accelerometers with 512 participants over the course of one year. We first determined whether IPLs sufficiently summarized (a) distributions of daily MVPA bout minutes and (b) time intervals between days where assigned MVPA goals were met. Second, we investigated whether markers of self-organization differed by study incentive arms (i.e., contingent, immediate versus noncontingent, delayed financial reinforcement), which tests the hypothesis that task incentives play a role in the self-organization process. Last, we examined IPLs summarizing behavior from the beginning of the year-long intervention and determined whether the features of these distributions predicted resistance to sustained performance of PA later in the trial. Because this is the first time these dynamical questions have been asked, it is difficult to make predictions, so this work should be considered exploratory. This point notwithstanding, we hypothesized that more stochastic PA habits at the outset of the study would be indicative of malleability that is conducive to sustained behavior change in response to an intervention. We anticipate that the results of this exploratory work will help determine how the structural organization of PA either hinders or facilitates targeted health behavior change efforts and the degree to which task-incentive networks may be involved in this process.

\section{METHODS}

\section{WalkIT Arizona Design}

WalkIT Arizona was a year-long clinical trial with 512 insufficiently active adults $(64.3 \%$ female, mean age $=45.5 \pm 9.1 \mathrm{yr}, 18.8 \%$ Hispanic, $84 \%$ White) from Maricopa County, Arizona (M. A. Adams et al., 2019). Participants were asked to wear an ActiGraph GT9X wrist-worn accelerometer during awake hours and to sync (i.e., upload) data from their accelerometer each day to project servers. Each day a new MVPA goal was provided via text message to participants (e.g. "Goal for $7 / 1$ is 30 
min”), and, after syncing with data servers, participants were informed of whether this goal was met, with select participants receiving financial reinforcement for achieving their goal. An automated, cloudbased system was designed for this study and online 24 hours/day, 365 days per year to receive and process accelerometer data, determine whether goals were met, calculate financial rewards, transmit text message feedback to participants, and send e-gift cards, when appropriate. A full description of the WalkIT Arizona methodology and sample characteristics is available in Adams et al (2019) and a brief summary is provided below.

Study recruitment primarily occurred through Facebook. Eligible participants were initially enrolled in a baseline phase, during which MVPA was passively recorded using a blinded accelerometer. After approximately 10 days in the baseline, each participant was enrolled into one of two reinforcement conditions: Immediate Reinforcement, where participants earned monetary rewards immediately upon meeting a daily goal and syncing their device by noon the next day or Delayed Reinforcement, where participants received a reward every 60 days if they wore their accelerometer for at least 10 hours on 4 out of the last 7 days. Similarly, participants were also enrolled into one of two goal type conditions: Static Goals, where the daily MVPA goal was set to 30 minutes or Adaptive Goals, where the goal was set to equal the 60th rank-order percentile based on moving window of MVPA minutes accrued over the previous nine days. The study was carried out as a $2 \times 2$ factorial design with participants blockrandomized into one of four groups defined by the reinforcement/goal combinations. Regardless of study group, after successfully meeting a daily goal and syncing their accelerometer, participants were provided a feedback text message with praise and their next goal (e.g., "Cheers, James! Goal met! 63 min yesterday. Goal for $7 / 2$ is $35 \mathrm{~min}$ ).

\section{Measures}

Daily MVPA: The number of MVPA bout minutes on a given day was calculated. Previous work (Freedson, P.S., Melanson, E., Sirard, 1998; Troiano et al., 2008) has outlined the specifics of defining bout minutes and establishing PA intensity.

Daily Goal Attainment Inter-response Time: We also considered the time interval (in days) between consecutive instances in which a participant met a daily goal and synced their device by noon 
the next day. By definition, the minimum time interval is one day. This measure is analogous to interresponse time (IRT) from behavior analysis; consequently, we refer to it as goal attainment IRT.

Inverse Power Law Features: Each participant's IPL features were calculated for both daily MVPA and goal attainment IRT over their first 100 days of study enrollment, excluding the baseline. This allowed us to quantify aspects of participant behavior at the beginning of their enrollment and to determine if features of this behavior were predictive of continued success in meeting intervention goals. This calculation was performed by creating a frequency table of each measure and fitting the following equation to this data:

$$
\log f=a+b \log x
$$

where $(x, f)$ pairs represent the frequency, $f$, of $x$ daily bout minutes/goal attainment IRTs recorded in the first 100 days. $a$ and $b$ are parameters that were estimated. Exponentiating both sides of this equation yields the IPL:

$$
f=C x^{b},
$$

where $C$ is the constant $e^{a} . b$ is known as the scaling exponent, which quantifies the "fatness" of the IPL tail while $C$ is associated with the relative frequency of smaller values in the distribution. The adjusted coefficient of determination $r^{2}$ for the fit is a measure of the degree to which the measures conformed to an IPL, with a penalty for larger numbers of predictors. See Fig. 2 for an illustration of the procedures for calculating IPL features.

\begin{tabular}{|c|c|}
\hline Goal Attainment IRT (days) $(x)$ & $\begin{array}{c}\text { Frequency } \\
(f)\end{array}$ \\
\hline 1 & 21 \\
\hline 2 & 7 \\
\hline 3 & 5 \\
\hline 4 & 5 \\
\hline 5 & 3 \\
\hline 7 & 1 \\
\hline 9 & 1 \\
\hline
\end{tabular}

$<$ INSERT FIGURE 2>

(Arranged as a $2 X 1$ row and joined $w /$ above table, which is part of the figure)

Figure 2: Illustration of the IPL fitting procedure for a representative individual. The table above the figure illustrates a frequency table for goal attainment IRT that will serve as the data for the IPL fit. In 
the bottom left figure, a linear function is fit to the log of the data. On the bottom right, these fitted parameters specify an IPL relationship when the raw data is plotted.

Goal Attainment: The primary outcome for regression analyses was long-term intervention performance, operationalized as the number of times after the 100th day in the intervention on which the daily goal was met and the accelerometer was synced by noon by the next day. This was denoted as $g_{>100}$. By focusing on intervention performance towards the end of the year-long intervention, we aimed to assess behavior most consistent with MVPA maintenance. Statistical analyses involving $g_{>100}$ were adjusted for the number of goals met over the first 100 days in the study, which was denoted as $g_{\leq 100}$.

Burstiness: The burstiness of goal attainment IRT was calculated. (For clarity, we note that burstiness cannot be calculated for daily MVPA since there is no 'event' threshold). Typically, burstiness is measured by the simple metric $\frac{\sigma-\mu}{\sigma+\mu}=\frac{r-1}{r+1}$, where $\mu$ and $\sigma$ are the mean and standard deviation of inter-event times and $r=\frac{\sigma}{\mu}$. However, this measure was developed for summaries of an infinite or very large number of events and, as such, it fails to consider the effects of a finite number of events. Kim and Jo (2016) addressed this shortcoming and proposed the following alternative measure of burstiness:

$$
B=\frac{(n-2)(r \sqrt{n+1}-(1-n \bar{y}) \sqrt{n-1})}{r(n \sqrt{n+1}-2(n-1))+(n-2 \sqrt{n+1}) \sqrt{n-1}(1-n \bar{y})}
$$

where $n$ is the number of unique goal attainment IRTs and $\bar{y}=\frac{y_{m}}{y_{d}}$, with $y_{m}$ equal to the minimum goal attainment IRT and $y_{d}$ equal to the duration of the time series. Burstiness values are expressed on a continuum, ranging from -1 (regularly spaced events) to 0 (stochastic events) to 1 (bursty events). Burstiness was calculated separately for goal attainment IRTs in first 100 days of the study and after the $100^{\text {th }}$ day of enrollment, denoted as $B_{\leq 100}$ and $B_{>100}$, respectively. Examples of bursty and non bursty patterns are shown in Fig. 3.

$<$ INSERT FIGURE 3>

Figure 3: Representative cases of low burstiness $(B=-0.11)$ and high burstiness $(B=0.82)$ in participants' goal attainment IRT. Each vertical line represents a day on which participants met their daily MVPA goal and synced their device by noon the following day. 
Covariates: The following covariates were included in the regression analyses outlined below. Design variables: census-block walkability (higher vs. lower), census-block socioeconomic status (SES; higher vs. lower). Demographic characteristics: ethnicity (white vs. any non-white ethnicity), marital status (married vs. not married [e.g., single, separated, divorced]), educational attainment (college graduate and higher vs. other), gender, number of children living in the household, and body mass index $\left(\mathrm{BMI}=\mathrm{kg} / \mathrm{m}^{2}\right)$.

\section{Sample Description}

The analytical sample criteria were based on valid wear days, defined as days on which at least six hours of accelerometer wear time was recorded, or the daily physical activity goal was met. Days not meeting these criteria were eliminated from all analyses. Participants who did not have at least 50 valid days both before and after their 100th day in the intervention were eliminated from analyses, leading to a sample size of 415 individuals. By definition, a participant who met $g$ goals had $g-1$ goal attainment IRTs; for small values of $g$, the IPLs fit to the small number goal attainment IRTs may not have been robust summaries of the data. To account for this scenario, an additional inclusion criterion was used for goal attainment IRTs, such that participants were required to have a minimum of five unique goal attainment IRT measures and to have met their goal on at least 10\% (i.e. 10) of days before their $100^{\text {th }}$ day of enrollment. This eliminated an additional 94 participants from the study, which led to a total sample size of 321. Demographics for this sample group are provided in Table 1.

\begin{tabular}{|c|c|c|}
\hline \multicolumn{3}{|c|}{ Quantitative Variables } \\
\hline & Mean & Std. Deviation \\
\hline Age & 45.9 & 9.1 \\
\hline BMI & 33.0 & 7.0 \\
\hline \# Children under 18 & 0.97 & 1.2 \\
\hline \multicolumn{3}{|c|}{ Categorical Variables } \\
\hline & Frequency & $\%$ \\
\hline Ethnicity - White & 301 & 72.5 \\
\hline Marital Status - Married & 284 & 68.4 \\
\hline Education - College Grad + & 282 & 68.0 \\
\hline Census-block walkability - High & 223 & 53.7 \\
\hline Census-block SES - High & 222 & 53.5 \\
\hline
\end{tabular}

Table 1: Descriptive statistics for demographics of the sample defined by the inclusion criteria for goal attainment IRTs $(N=321)$. 


\section{Statistical Analyses}

Summary Statistics: The appropriateness of using an IPL to summarize participants' a.) daily minutes of MVPA bouts and b.) goal attainment IRT was assessed by examining the distributions of adjusted $r^{2}$ values for each of these variables over all participants meeting the inclusion criteria. Descriptive statistics for all independent/dependent variables were calculated. Correlations between burstiness and goal attainment in the first 100 days of enrollment $\left(B_{\leq 100}\right.$ and $\left.g_{\leq 100}\right)$ and after the $100^{\text {th }}$ day $\left(g_{>100}\right.$ and $\left.B_{>100}\right)$ were also calculated as was the correlation in burstiness during these two time periods $\left(B_{\leq 100}\right.$ and $\left.B_{>100}\right)$.

Burstiness and Reward Timing: To test the hypothesis that burstiness in the goal attainment IRT was associated with immediate rewards, which can be viewed as a process that induces task prioritization, the following regression model was fit to the data:

$$
B_{i j}=\alpha+\beta_{1} t_{j}+\beta_{2} r_{i}+\sum_{m=1}^{M} \gamma_{m} z_{m i}+u_{i}
$$

where $b_{i j}$ is the burstiness for individual $i$ recorded at measurement occasion $j=1$ or 2 , corresponding to $B_{\leq 100}$ and $B_{>100} . t_{j}$ is the effect of the measurement occasion ( $\left.\leq 100 \mathrm{vs.}>100\right), r_{i}$ is the reward timing (Immediate vs. Delayed) for individual $i, z_{m i}$ are the remaining covariates for individual $i$, and $u_{i}$ is an individual-level intercept. $r_{i}$ could have been incorporated into the $z_{m i}$ summation term, but we chose to show it on its own since this is our main variable of interest in assessing task prioritization. The individual level intercept $\left(u_{i}\right)$ is often accounted for by a random-intercept, mixed effects hierarchicat linear model approach, but because nearly all covariates are time invariant, this approach makes the strong assumption that covariates are uncorrelated with all unobserved variables. To avoid this assumption we follow the example of Plümper and Troeger (2007) and decompose the random effects into explained and unexplained sources before running a fixed-effects model. To determine effect-size, Cohen's $d$ was calculated by dividing the difference in the estimated marginal means for the Immediate and Delayed groups by the pooled standard deviation.

Burstiness and Success Meeting MVPA Goals: To determine the factors associated with sustained success in meeting goals throughout enrollment in the study, $g_{>100}$ was regressed on $B_{\leq 100}$, $B_{>100}$, and $g_{\leq 100}$ in both simple linear regression and multiple regression models. An additional model included a $B_{\leq 100}$-by- $g_{\leq 100}$ interaction term to assess if burstiness in goal attainment early in the trial 
had a differential effect based on participants' early success in meeting daily MVPA goals. To further explore the $B_{\leq 100}$-by- $g_{\leq 100}$ interaction, the data was stratified into four groups defined by the quartiles of $g_{\leq 100}$ and a distinct multiple regression model was calculated for each quartile group. Demographic covariates were included in all regression models. Additionally, since previous research with IPLs has noted the effects of the fit index $r^{2}$ and shape parameter $b$ on outcomes, all non-stratified regression analyses were re-run with these values included. Table 2 illustrates the components of the regression models fit to the data.

\section{Burstiness and Reward Timing}

Dependent Variables: Burstiness ( $B_{\leq 100}$ and $B_{>100}$ )

Independent Variables: Reward timing (immediate vs. delayed), measurement interval ( $>100$ Days vs. $\leq 100$ Days) Covariates: Goal type, census-block walkability, census-block SES, ethnicity, marital status, education, gender, \# of children living in the household, BMI

Methodology: $B_{\leq 100}$ and $B_{>100}$ clustered by individual in a modified fixed effects model (see Burstiness and Reward Timing section of Statistical Analyses for details)
Burstiness and Success Meeting MVPA Goals

Dependent Variable: $g_{>} 00$

Independent Variables: $g_{\leq 100}, B_{\leq 100}, B_{>100}$

Covariates: Reward timing, goal type, census-block walkability, census-block SES, ethnicity, marital status, education, gender, \# of children living in the household, BMI Methodology: i.) Bivariate regression of DV on each IV; ii.) Multiple Regression; iii.) Multiple Regression with $g_{\leq 100} *$ $B_{\leq 100}$ interaction

Table 2: Components of the Burstiness and Reward Timing and Burstiness and Success Meeting MVPA Goals regression approaches.

Nonlinear Effects \& Sensitivity Analysis: The regression models described above both assume that all effects are linear and additive, which is likely to be violated. Supplementary analysis with quantile regression (results not shown) yielded qualitatively similar results to those outlined below. Furthermore, while preliminary analysis indicated that 100 days was a reasonable selection as a stratification point between predictor and outcome measures, this choice was somewhat arbitrary. To test the sensitivity of findings to this parameter, we performed a sensitivity analysis that is fully detailed in Appendix A

\section{RESULTS}

Summary Statistics: Table 3 provides descriptive statistics and Fig. 4 illustrates the distribution of adjusted $r^{2}$ values for the IPL fit over all participants. The adjusted $r^{2}$ values for daily bouts of MVPA were relatively small, indicating that IPLs do not appear to be an appropriate summarization of this feature. Goal attainment IRTs were much better summarized by IPLs and as a result, all subsequent analyses solely focused on this measure. The burstiness of participants' goal attainment IRTs in both the first 100 days $\left(B_{\leq 100}\right)$ and beyond the first 100 days $\left(B_{>100}\right)$ are also summarized in Table 3 and Fig. 4. The values ranged from approximately 0 to approximately 1 , meaning that participants' 
occurrences of meeting goals roughly varied from stochastic through bursty, but did not extend to regular patterns (which would have been indicated by $B$ approaching -1).

\begin{tabular}{|c|c|c|c|c|}
\hline & $\begin{array}{c}\text { Mean } \\
(\text { SD) }\end{array}$ & Min/Max & Median & IQR \\
\hline $\begin{array}{c}\text { IPL Adjusted } r^{2} \text { (daily } \\
\text { MVPA bout minutes) }\end{array}$ & $0.30(0.25)$ & $-0.04 / 0.91$ & 0.27 & 0.46 \\
\hline $\begin{array}{c}\text { IPL Adjusted } r^{2} \text { (goal } \\
\text { attainment IRTs) }\end{array}$ & $0.69(0.24)$ & $-0.20 / 0.99$ & 0.75 & 0.27 \\
\hline $\begin{array}{c}\text { IPL scaling exponent } \boldsymbol{b} \\
\text { (goal attainment IRTs) }\end{array}$ & $-1.06(0.51)$ & $-2.63 / 0.05$ & -1.02 & 0.68 \\
\hline$g_{\leq 100}$ & $\mathbf{- 1 . 4 0 ( 0 . 4 2 )}$ & $\mathbf{- 2 . 6 3 / - 0 . 4 5}$ & $\mathbf{- 1 . 3 6}$ & $\mathbf{0 . 5 4}$ \\
\hline$g_{>100}$ & $66.1(41.4)$ & $10 / 77$ & 35 & 21 \\
\hline$B_{\leq 100}$ & $0.32(0.17)$ & $-0.11 / 0.82$ & 0.32 & 57 \\
\hline$B_{>100}$ & $0.28(0.19)$ & $-0.41 / 1.00$ & 0.26 & 0.21 \\
\hline
\end{tabular}

Table 3: Descriptive statistics for regression variables. The inclusion criteria for goal attainment IRTs was used to define the sample for the calculation of all statistics except adjusted $r^{2}$ for daily bout minutes. ${ }^{+}$indicates that the bottom, bold values are scaling exponent summary statistics for only those individuals with an adjusted $r^{2}>0.8$ (n=125). This was done since the scaling exponent is valid/interesting only when distributions are reasonably approximated by IPLs.

\section{$<$ INSERT FIGURE 4> \\ (Arranged as $2 \times 2$ Matrix)}

Figure 4: The distribution of adjusted $r^{2}$ values for IPL fits of daily minutes of MVPA bouts (top left) and goal attainment IRTs (top right). The distribution of $B_{\leq 100}$ and $B_{>100}$ values for goals met are shown in the bottom left and right rows, respectively.

Burstiness and Reward Timing: Table 4 illustrates the results of the hierarchical linear model built to test the hypothesis that burstiness is associated with task prioritization and therefore would be higher in the Immediate (versus Delayed) reinforcement arm of the trial. The results confirmed this hypothesis and indicated that burstiness was significantly higher for Immediate versus Delayed reinforcement. Estimated marginal means were then calculated for these two arms, which were 0.339 for the Immediate arm and 0.262 for the Delayed arm. Cohen's $d$ for this difference was 0.43 , which reflects a small-to-moderate effect. The model also indicated that burstiness was significantly lower for individuals from low SES block groups, males and when calculated after the $100^{\text {th }}$ day of enrollment as opposed to before.

\begin{tabular}{|l|c|c|c|}
\hline & $\beta$ & $S E$ & $p$ \\
\hline Reward Timing (Immediate vs. Delayed) & 0.077 & 0.009 & $<\mathbf{0 . 0 0 1}$ \\
\hline
\end{tabular}




\begin{tabular}{|c|c|c|c|}
\hline Goal Type (Static vs. Adaptive) & 0.014 & 0.009 & 0.151 \\
\hline Measurement Interval ( $>100$ Days $v s . \leq 100$ Days) & -0.048 & 0.009 & $<0.001$ \\
\hline Neighborhood Walkability ( $\underline{\text { Low }} v$ s. High) & 0.0003 & 0.009 & 0.973 \\
\hline Neighborhood SES (Low vs. High) & -0.020 & 0.010 & 0.042 \\
\hline Age* & -0.0002 & 0.001 & 0.729 \\
\hline Sex (Male vs. Female) & -0.048 & 0.010 & $<0.001$ \\
\hline Marital Status (Not Married vs. Married) & -0.004 & 0.011 & 0.730 \\
\hline Ethnicity (White vs. Other) & -0.015 & 0.011 & 0.146 \\
\hline Education ( College Grad + vs. $<$ College Grad) & -0.018 & 0.010 & 0.070 \\
\hline \# of Children in Home* & -0.007 & 0.004 & 0.079 \\
\hline BMI* & 0.0004 & 0.001 & 0.591 \\
\hline
\end{tabular}

Table 4: Results of modified fixed effects model to assess the effect of reward timing and covariates on the burstiness of goal attainment IRTs. A * indicates a continuous variable and for non-continuous (i.e., qualitative) variables, the levels follow each variable with the referent level underlined. $p$ values less than 0.05 are shown in bold.

Burstiness and Success Meeting MVPA Goals: The correlation between $B_{\leq 100}$ and $B_{>100}$, was 0.25 , indicating a considerable variation in burstiness between these two intervals. The relationships between burstiness and goals met during these two intervals were not consistent, with a correlation between $B_{\leq 100}$ and $g_{\leq 100}$ of 0.43 and a correlation between $B_{>100}$ and $g_{>100}$ of 0.09 , suggesting that that burstiness and concurrent success in meeting MVPA goals are more closely linked earlier in the trial.

Table 5 illustrates the results of the three regression models that examined the effect of IPL features on $g_{>100}$. In bivariate models, $g_{\leq 100}$ and $B_{\leq 100}$ were both significantly associated with higher values of $g_{>100}$, but $B_{>100}$ was not. After controlling for each other, the relationship between $g_{\leq 100}$ and $g_{>100}$ remained similar, but the positive association between $B_{\leq 100}$ and $g_{>100}$ reversed, with greater burstiness values now associated with smaller $g_{>100}$, at a level approaching significance.

When an interaction term between $g_{\leq 100}$ and $B_{\leq 100}$ was included, $g_{\leq 100}$ had a larger effect on $g_{>100}$, but $B_{\leq 100}$ lost its trend towards significance. The interaction term neared significance such that high burstiness before day 100 was more strongly associated with fewer goals met later in the intervention for individuals meeting a relatively large number of goals before day 100. To further illustrate this point, multiple regression models, stratified by quartiles of $g_{\leq 100}$ were fit to the data. Figure 5 illustrates the $B_{\leq 100}$ versus $g_{>100}$ relationship for the different quartiles of $g_{\leq 100}$, with all other 
covariates set equal to their means. A statistically significant association between burstiness and goals attained after day 100 was present only for individuals in the fourth quartile of $g_{\leq 100}$, i.e. those who were most successful in meeting goals over the first 100 days of the intervention. The $B_{\leq 100}$ coefficient for this group was -101.7 .

\begin{tabular}{|c|c|c|c|c|c|c|c|c|c|}
\hline & \multicolumn{3}{|c|}{ Bivariate Models } & \multicolumn{3}{c|}{$\begin{array}{c}\text { Multiple Regression } \\
\text { Model }\end{array}$} & \multicolumn{3}{c|}{ Interaction Model } \\
\hline & $\beta(S E)$ & $\hat{\beta}$ & $p$ & $\beta(S E)$ & $\hat{\beta}$ & $p$ & $\beta(S E)$ & $\hat{\beta}$ & $p$ \\
\hline$g_{\leq 100}$ & $\begin{array}{c}1.65 \\
(0.12)\end{array}$ & 0.62 & $<\mathbf{0 . 0 0 1}$ & $\begin{array}{c}1.71 \\
(0.13)\end{array}$ & 0.65 & $<\mathbf{0 . 0 0 1}$ & $\begin{array}{c}2.16 \\
(0.29)\end{array}$ & 0.82 & $<\mathbf{0 . 0 0 1}$ \\
\hline$B_{\leq 100}$ & $\begin{array}{c}40.02 \\
(13.81)\end{array}$ & 0.16 & $\mathbf{0 . 0 0 4}$ & $\begin{array}{c}-21.11 \\
(12.11)\end{array}$ & -0.09 & 0.08 & $\begin{array}{c}12.88 \\
(23.17)\end{array}$ & 0.05 & 0.58 \\
\hline$B_{>100}$ & $\begin{array}{c}4.97 \\
(12.61)\end{array}$ & 0.02 & 0.88 & $\begin{array}{c}1.60 \\
(10.19)\end{array}$ & 0.007 & 0.87 & $\begin{array}{c}2.44 \\
(10.17)\end{array}$ & 0.01 & 0.81 \\
\hline $\begin{array}{l}g_{\leq 100} \\
* B_{\leq 100}\end{array}$ & - & - & - & - & - & - & $\begin{array}{c}-1.10 \\
(0.64)\end{array}$ & -0.41 & 0.09 \\
\hline
\end{tabular}

Table 5: Regression results with goals met after $100^{\text {th }}$ day of enrollment $\left(g_{>100}\right)$ as the dependent variable. $g_{\leq 100}=$ number of goals met during the first 100 days of enrollment, $B_{\leq 100}=$ burstiness during first 100 days of enrollment, $B_{>100}=$ burstiness during remainder of enrollment, $\beta(S E)=$ regression coefficient (standard error), and $\hat{\beta}=$ standardized regression coefficient. $p$-values $<0.05$ are shown in bold.

\section{$<$ INSERT FIGURE 5>}

Figure 5: Results of quartile regression. $B_{\leq 100}$ versus $g_{>100}$ stratified by $g_{\leq 100}$ quartiles.

When introducing $r^{2}$ and $b$ into these analyses, there was a statistically significant relationship between each of these terms and $g_{>100}$ in bivariate regression models. But this was not the case for the multiple regression and interaction models, where their inclusion minimally changed the results. Therefore, the results for the most parsimonious IPL model was presented in this section.

\section{DISCUSSION}

Our central finding was that stochasticity in meeting goals at the outset of a PA intervention was associated with greater malleability of behavior, while burstiness was associated with resistance to change during the trial. Quartile regression indicated that this phenomenon occurred specifically for participants who were successful at meeting goals early in the intervention. In other words, greater goal attainment early in the intervention was more strongly associated with later success when the patterns of that early goal attainment were relatively stochastic (i.e., less bursty). When early goal attainment 
was highly clustered in time, goal attainment after 100 days was relatively poor. For individuals meeting fewer goals early in the intervention, burstiness did not predict later goal attainment, which may suggest that, for individuals that are less responsive to the intervention, meeting goals in any capacity at the beginning of treatment, regardless of the temporal pattern, is predictive of later success. That is, low baseline MVPA was the primary obstacle to sustained intervention success, with established habits (as revealed by greater burstiness) providing headwinds for only those subjects who best responded to treatment.

For practitioners, the results may be understood most intuitively within the context of a "breaking down" prior habits. As an extreme example, one may consider the strategies of a military drill sergeant, who prescribes PA at random intervals during basic training. While this level of "breaking down" is certainly over-kill for standard studies, mHealth interventions may be more effective if PA prompts are more stochastic early in a behavioral intervention, particularly for those who show high levels of engagement. More broadly, stochasticity may be a sign of openness or readiness to change PA patterns and has been identified in other work as a critical process for PA adoption (Iso-ahola, 2011). Inducing greater stochasticity may provide a "softening up" in preparation for a lifestyle transformation in one's established PA habits.

Our results also revealed that immediate, PA contingent rewards versus delayed, non-contingent rewards were associated with higher levels of burstiness in PA goal attainment, which is consistent with previous assertions of burstiness as consequence of task prioritization. By definition, the burstiness engendered by immediate rewards comes at the expense of stochasticity. If stochasticity is indeed associated with breaking down old habits, then immediate rewards may inhibit this effect by freezing counter-productive habits in place, unless rewards are specifically aimed at generating stochastic PA. Behavioral literature suggests that discontinuing reinforcement increases variability in behavior (Skinner, 1953). Therefore, an effective intervention strategy, particularly for people successfully meeting PA goals, may be to initially provide incentives, but then eliminate them to allow goal achievement to become more stochastic. If these hypotheses prove correct, burstiness may be used as a relatively simple dynamical index that can be used to tailor feedback for individuals attempting to change their behavior. For example, participants who enter an intervention with relatively bursty PA 
patterns may be informed that rewards will be temporarily delayed while they are encouraged to explore a greater variety of opportunities for PA within a broader range of life contexts - for example, walking or stair climbing during the work day rather than exclusively after work or on weekends.

There are several implications for measurement of self-organization in daily PA that arise from the current results. IPLs were observed for some participants with respect to daily MVPA bouts, with the largest fits having adjusted $r^{2}$ values near 0.75 . But the mode was zero, indicating that IPLs may not be the best model to use in general for PA magnitude. On the other hand, IPLs were far more common for goal attainment IRTs, clustering at the higher range of adjusted $r^{2}$ with a mode near 0.9 . Fit index, $r^{2}$, and shape parameter, $b$, were significantly related to $g_{>100}$ in bivariate regression models, but not within the multiple regression and interaction models. As such, the most parsimonious model included burstiness of goals met within the first 100 days of the intervention. A full spectrum of burstiness was observed within this sample, ranging from stochastic (at the low end) to highly clustered at the high end, within a Gaussian-type distribution with a mode near 0.3. This variance in burstiness may have played some role in its power to predict malleability above and beyond the strong effect of $g_{>100}$. Yet many other factors could also have lent power to burstiness, including its embeddedness in time compared to IPLs, or factors unique to this sample or to the selection of daily PA as an outcome measure. These results stand in minor contrast to other studies involving different types of PA, in which direct IPL features were used as predictors, and may serve to integrate the PA literature with the literature on non-PA behaviors (e.g., flows of work tasks). Future studies may wish to continue to examine both IPLs and burstiness as indicators of the self-organization of habits.

This study had several limitations. It was designed to be exploratory due to the novelty of the design within the context of a PA intervention, so replication and extension are needed before applying the current results with confidence to future interventions. Over the course of a one-year trial, most participants did not wear the accelerometer every day, so the data used in these analyses likely exclude MVPA that was performed, but not measured. Regression effect sizes were small and notable inferential statistical tests tended towards, but did not meet, significance at $\alpha=0.05$. There is also the potential for over-interpretation of results as self-organization without robust IPLs in both temporal and bout-size dynamics. This is especially true since our sample size and measurement periods were relatively small 
and concerns over the accuracy of the log-log fitting routine we used have been raised (Clauset, Shalizi, \& Newman, 2009). Similarly, less constrained regression approaches that model non-linear associations among covariates may affect findings and should be explored in future work. Additionally, the notion of incentives and task prioritization were not tested directly, and so should be interpreted with caution.

Most broadly, the current results support the importance of ongoing research that moves beyond the identification of static mean targets. For decades, PA research has shown us where to aim intervention efforts (e.g., 150 minutes per week). But only recently have we gained access to mobile data that can show us how best to get there. Ideally, this data will allow us to better tailor treatments to individuals and their life situations, as each unfolds in time.

\section{FUNDING}

This work was supported by the National Cancer Institute at the National Institutes of Health [XXXXXXXXX]. The funding agency was not involved in any aspect of this study or manuscript.

\section{ACKNOWLEDGEMENTS}

The authors acknowledge the support of XXXXXXX and XXXXXXX, along with many undergraduate and graduate research assistants helping with data collection.

\section{REFERENCES}

Adams, M. A., Hurley, J. C., Phillips, C. B., Todd, M., Angadi, S. S., Berardi, V., ... Hooker, S. (2019). Rationale, design, and baseline characteristics of WalkIT Arizona: A factorial randomized trial testing adaptive goals and financial reinforcement to increase walking across higher and lower walkable neighborhoods. Contemporary Clinical Trials, 81, 87-101. https://doi.org/10.1016/j.cct.2019.05.001

Adams, M. A., Hurley, J. C., Todd, M., Bhuiyan, N., Jarrett, C. L., Tucker, W. J., ... Angadi, S. S. (2017). Adaptive goal setting and financial incentives: a $2 \times 2$ factorial randomized controlled trial to increase adults' physical activity. BMC Public Health, 17(1), 286. https://doi.org/10.1186/s12889-017-4197-8

Adams, M., Hurley, J., Phillips, C., Todd, M., Berardi, V., Hovell, M., \& Hooker, S. (2019). Rationale, Design, and Baseline Characteristics of WalkIT Arizona: A Factorial Randomized Trial Testing Adaptive Goals and Financial Reinforcement to Increase Walking across Higher 
and Lower Walkable Neighborhoods. Contemporary Clinical Trials, (In Press).

Barabási, A. L. (2005). The origin of bursts and heavy tails in human dynamics. Nature, 435(7039), 207-211. https://doi.org/10.1038/nature03459

Bellettiere, J., Winkler, E. A. H., Chastin, S. F. M., Kerr, J., Owen, N., Dunstan, D. W., \& Healy, G. N. (2017). Associations of sitting accumulation patterns with cardio-metabolic risk biomarkers in Australian adults. PLoS ONE, 12(6). https://doi.org/10.1371/journal.pone.0180119

Benjamin, E. J., Muntner, P., Alonso, A., Bittencourt, M. S., Callaway, C. W., Carson, A. P., ... Virani, S. S. (2019). Heart Disease and Stroke Statistics-2019 Update: A Report From the American Heart Association. Circulation, 139(10), e56-e528. https://doi.org/10.1161/CIR.0000000000000659

Blackwell, D. L., \& Clarke, T. C. (2016). Occupational differences among employed adults who met 2008 federal guidelines for both aerobic and muscle-strengthening activities: United States, 2008-2014. National Health Statistics Reports, 2016(94).

Brownson, R. C., Boehmer, T. K., \& Luke, D. A. (2005). Declining Rates of Physical Activity in the United States: What Are the Contributors? Annual Review of Public Health, 26(1), 421-443. https://doi.org/10.1146/annurev.publhealth.26.021304.144437

Corral, Á. (2003). Local distributions and rate fluctuations in a unified scaling law for earthquakes. Physical Review E - Statistical Physics, Plasmas, Fluids, and Related Interdisciplinary Topics, 68(3), 4. https://doi.org/10.1103/PhysRevE.68.035102

Cutt, H. E., Knuiman, M. W., \& Giles-Corti, B. (2007). Does getting a dog increase recreational walking? International Journal of Behavioral Nutrition and Physical Activity, 5(17).

Dave, S., Brothers, T. A., \& Swaab, T. Y. (2018). 1/f neural noise and electrophysiological indices of contextual prediction in aging. Brain Research, 1691, 34-43. https://doi.org/10.1016/j.brainres.2018.04.007

Delvenne, J. C., Lambiotte, R., \& Rocha, L. E. C. (2015). Diffusion on networked systems is a question of time or structure. Nature Communications, 6. https://doi.org/10.1038/ncomms8366

Dishman, R. K., \& Buckworth, J. (1996). Increasing physical activity: a quantitative synthesis. Medicine \& Science in Sports \& Exercise, 28(6), 706-719. Retrieved from 
https://journals.lww.com/nursing/00005768-199606000-00010.fulltext

Eckmann, J. P., Moses, E., \& Sergi, D. (2004). Entropy of dialogues creates coherent structures in email traffic. Proceedings of the National Academy of Sciences of the United States of America, 101(40), 14333-14337. https://doi.org/10.1073/pnas.0405728101

Ferster, C. B., \& Skinner, B. F. (1957). Schedules of reinforcement. East Norwalk: Appleton-CenturyCrofts. https://doi.org/10.1037/10627-000

Freedson, P.S., Melanson, E., Sirard, J. (1998). Calibration of the Computer Science and Applications, Inc. accelerometer. Medicine \& Science in Sports \& Exercise, 30(5), 777-781.

Gandica, Y., Carvalho, J., Aidos, F. S. Dos, Lambiotte, R., \& Carletti, T. (2016). On the origin of burstiness in human behavior: The wikipedia edits case, 1-12. Retrieved from http://arxiv.org/abs/1601.00864

Goldberger, A. L. (2006). Complex systems. In Proceedings of the American Thoracic Society (Vol. 3, pp. 467-471). https://doi.org/10.1513/pats.200603-028MS

Golding, I., Paulsson, J., Zawilski, S. M., \& Cox, E. C. (2005). Real-time kinetics of gene activity in individual bacteria. Cell, 123(6), 1025-1036. https://doi.org/10.1016/j.cell.2005.09.031

Grol, R. (2001). Successes and Failures in the Implementation of Evidence-Based Guidelines for Clinical Practice. Medical Care, 39(8), 46-54.

Iribarren, J. L., \& Moro, E. (2011). Branching dynamics of viral information spreading. Physical Review E - Statistical, Nonlinear, and Soft Matter Physics, 84(4). https://doi.org/10.1103/PhysRevE.84.046116

Iso-ahola, S. E. (2011). Conscious-Nonconscious Processing Explains Why Some People Exercise but Most Don't. Journal of Nature and Science, 3(6), 1-16.

Jo, H.-H., Karsai, M. M., Kertész, M., \& Kaski, K. (2012). Circadian pattern and burstiness in mobile phone communication. New Journal of Physics, 14(1), 013055.

Jo, H. H., Perotti, J. I., Kaski, K., \& Kertész, J. (2014). Analytically solvable model of spreading dynamics with Non-Poissonian processes. Physical Review X, 4(1), 1-6. https://doi.org/10.1103/PhysRevX.4.011041

Karsai, M., Kivelä, M., Pan, R. K., Kaski, K., Kertész, J., Barabási, A. L., \& Saramäki, J. (2011). 
Small but slow world: How network topology and burstiness slow down spreading. Physical Review E - Statistical, Nonlinear, and Soft Matter Physics, 83(2), 1-4. https://doi.org/10.1103/PhysRevE.83.025102

Kim, E. K., \& Jo, H. H. (2016). Measuring burstiness for finite event sequences. Physical Review E, 94(3), 032311.

Kauffmann, S. (1995). Chaos, Complexity, Self-Organisation in Nature and Society. New York, NY: Oxford University Press.

Kello, C. T., Anderson, G. G., Holden, J. G., \& Van Orden, G. C. (2008). The pervasiveness of 1/f scaling in speech reflects the metastable basis of cognition. Cognitive Science, 32(7), 1217-1231. https://doi.org/10.1080/03640210801944898

Kruger, J., Ham, S., \& Kohl III, H. (2005). Trends in leisure-time physical inactivity by age, sex, and race/ethnicity-United States, 1994-2004. MMWR MorbMortalWklyRep, 54(39), 991-994.

Lee, J.-H., Goh, S., Kim, S. H., Kim, J. W., Lee, Y. J., Kim, S. J., \& Choi, M. (2019). Statistical properties of human activity and criticality in active behavior. EPL (Europhysics Letters), 126(6), 68001. https://doi.org/10.1209/0295-5075/126/68001

Malmgren, R. D., Stouffer, D. B., Motter, A. E., \& Amaral, L. A. N. (2008). A Poissonian explanation for heavy tails in e-mail communication. Proceedings of the National Academy of Sciences of the United States of America, 105(47), 18153-18158. https://doi.org/10.1073/pnas.0800332105

Marcus, B. H., Forsyth, L. H., Stone, E. J., Dubbert, P. M., McKenzie, T. L., Dunn, A. L., \& Blair, S. N. (2000). Physical activity behavior change: Issues in adoption and maintenance. Health Psychology, 19(1, Suppl), 32-41. https://doi.org/10.1037/0278-6133.19.suppl1.32

Nakamura, T., Takumi, T., Takano, A., Aoyagi, N., Yoshiuchi, K., Struzik, Z. R., \& Yamamoto, Y. (2008). Of mice and men - Universality and breakdown of behavioral organization. PLoS ONE, 3(4). https://doi.org/10.1371/journal.pone.0002050

Paraschiv-Ionescu, A., Buchser, E., \& Aminian, K. (2013). Unraveling dynamics of human physical activity patterns in chronic pain conditions. Scientific Reports, 3(1), 1-10. https://doi.org/10.1038/srep02019

Pincus, D. (2014). One bad apple: Experimental effects of psychological conflict on social resilience. 
Interface Focus, 4(5). https://doi.org/10.1098/rsfs.2014.0003

Pincus, D., Cadsky, O., Berardi, V., Asuncion, C. M., \& Wann, K. (2019). Fractal Self-Structure and Psychological Resilience. Nonlinear Dynamics, Psychology, and Life Sciences, 23(1), 57-78.

Pincus, D., Eberle, K., Walder, C. S., Kemp, A. S., Lenjav, M., \& Sandman, C. A. (2014). The role of self-injury in behavioral flexibility and resilience. Nonlinear Dynamics, Psychology, and Life Sciences, 18(3), 277-296.

Pincus, D., \& Metten, A. (2010). Nonlinear Dynamics in Biopsychosocial Resilience. Nonlinear Dynamics, Psychology, and Life Sciences, 14(4), 353-380. Retrieved from https://www.researchgate.net/publication/47298239

Silverman, K., Higgins, S. T., Brooner, R. K., Montoya, I. D., Cone, E. J., Schuster, C. R., \& Preston, K. L. (1996). Sustained Cocaine Abstinence in Methadone Maintenance Patients Through Voucher-Based Reinforcement Therapy. Archives of General Psychiatry, 53, 409-415.

Skinner, B. (1953). Science and human behavior. New York, NY: Macmillan.

Sorribes, A., Armendariz, B. G., Lopez-Pigozzi, D., Murga, C., \& de Polavieja, G. G. (2011). The origin of behavioral bursts in decision-making circuitry. PLoS Computational Biology, 7(6). https://doi.org/10.1371/journal.pcbi.1002075

Starnini, M., Baronchelli, A., Barrat, A., \& Pastor-Satorras, R. (2012). Random walks on temporal networks. Physical Review E - Statistical, Nonlinear, and Soft Matter Physics, 85(5), 1-14. https://doi.org/10.1103/PhysRevE.85.056115

Troiano, R. P., Berrigan, D., Dodd, K. W., Masse, L. C., Tilert, T., \& McDowell, M. (2008). Physical Activity in the United States Measured by Accelerometer. Medicine \& Science in Sports \& Exercise, 40(1), 181-188. https://doi.org/10.1249/mss.0b013e31815a51b3

U.S. Department of Health and Human Services. (2018). Physical Activity Guidelines for Americans, 2nd edition.

Vazquez, A., Rácz, B., Lukács, A., \& Barabási, A. L. (2007). Impact of non-poissonian activity patterns on spreading processes. Physical Review Letters, 98(15), 2-5. https://doi.org/10.1103/PhysRevLett.98.158702

Ward, B. W., Clarke, T. C., Nugent, C. N., \& Schiller, J. S. (2015). Early Release of Selected 
Estimates Based on Data From the 2015 National Health Interview Survey. Retrieved from http://www.bobmorrison.org/wp-content/uploads/2017/01/cdc-report-on-uninsured-and-otherpopulation-stats.pdf

Williams, D. M., Lewis, B. A., Dunsiger, S., Whiteley, J. A., Papandonatos, G. D., Napolitano, M. A., ... Marcus, B. H. (2008). Comparing psychosocial predictors of physical activity adoption and maintenance. Annals of Behavioral Medicine, 36(2), 186-194. https://doi.org/10.1007/s12160008-9054-7

Wong, A. E., Vallacher, R. R., \& Nowak, A. (2016). Intrinsic dynamics of state self-esteem: The role of self-concept clarity. Personality and Individual Differences, 100, 167-172. https://doi.org/10.1016/j.paid.2016.05.024

Zhang, W., Schwenk, M., Mellone, S., Paraschiv-Ionescu, A., Vereijken, B., Pijnappels, M., ... Aminian, K. (2018). Complexity of Daily Physical Activity Is More Sensitive Than Conventional Metrics to Assess Functional Change in Younger Older Adults. Sensors, 18(7), 2032. https://doi.org/10.3390/s18072032 


\section{APPENDIX A: SENSITIVITY ANALYSIS}

A sensitivity analysis was performed to test the robustness of findings to changes in the stratification point between the predictor and outcome measures, which was set to 100 days in the analyses within this manuscript. This was performed by re-calculating predictor and outcome variables for stratification points ranging from 50 to 150 days in 10-day intervals (i.e. $50,60, \ldots, 140,150$ days) and re-fitting the multiple regression models described in the Burstiness and Success Meeting MVPA Goals section of the Statistical Methods section for each scenario. Figure 6 shows the results of these analyses. Regression coefficients are qualitatively similar for delineation points ranging from approximately 80 through 140 days. With the exception of a small uptick at 90 days, $p$-values were also qualitatively similar for delineation points ranging from 80 through 140 days. These results indicate that the negative association between burstiness and $g_{>100}$ found when using a delineation point of 100 days (summarized in Table 5) is a robust funding and qualitatively similar results would be found for a large range of delineation points. They also indicate that the interaction results approaching significance in Table 5 can be assumed to reflect actual trends.

\footnotetext{
$<$ INSERT FIGURE 6>

(Arranged as a $2 \times 1$ row)
}

Figure 6: Results of regression analyses when considering alternate delineation points as the boundary between predictor and outcome variables. The left figure illustrates the regression coefficient for $B_{\leq 100}$ in the multiple regression model $(M-B), B_{\leq 100}$ in the interaction model (I-B), and the $g_{\leq 100} * B_{\leq 100}$ interaction coefficient (I-I) for various stratification points. The right figure illustrates the p-value for these terms and has dashed lines at $\alpha=0.05$ and 0.1. 